\title{
Frailty and cognitive performance in older adults living in the community: a cross-sectional study
}

\author{
DaiEne de Morais FABRícIO ${ }^{1}$ \\ https://orcid.org/0000-0001-8609-1190
}

TiAGO DA SILVA ALEXANDRE2 2

MARCOS HoRTES NisIHARA CHAGAS 1,2

1 Department of Psychology, Federal University of São Carlos, São Carlos, SP, Brazil.

2 Department of Gerontology, Federal University of São Carlos, São Carlos, SP, Brazil.

Institution where the study was conducted: Federal University of São Carlos (UFSCar), São Carlos, SP, Brazil.

Received: $11 / 29 / 2018$ - Accepted: 08/12/2019

DOI: 10.1590/0101-60830000000216

\begin{abstract}
Background: Cognitive impairment and frailty are important problems affecting the elderly population. Frail elderly present worse overall cognitive performance. Objective: The aim of this study was to investigate general and domain-specific cognitive performance among non-frail, pre-frail, and frail elderly persons. Methods: This is a cross-sectional study in which 267 elderly persons living in São Carlos, SP were divided into three groups according to the frailty criteria defined by Fried et al. Cognitive performance was evaluated with a battery of cognitive tests covering domains such as memory, attention, language, and executive functioning. A multinomial logistic regression analysis adjusted for age, gender, and education was performed to evaluate the association between performance in cognitive domains and levels of frailty. Results: Frailty was significantly associated with lower scores on the global cognitive test (RRR $=0.86$; IC $95 \% 0.78$ $0.96 ; p<0.01)$, word list memory ( $R R R=0.92 ;$ IC $95 \% 0.86-0.99 ; p=0.02)$, and figure list recognition $(R R R=0.78 ;$ IC $95 \% 0.62-0.99 ; p=0.04)$. Pre-frailty was associated with lower scores on the word list memory $(R R R=0.92$; IC 95\% 0.86-1.00; $p=0.04)$ and naming test $(R R R=0.82 ;$ IC 95\% 0.69-0.99; $p=0.03)$. Discussion: Frailty syndrome can influence general cognition and specific domains such as memory and language. Prospective studies will be fundamental to evaluate the causal relation between frailty and cognition.
\end{abstract}

Fabrício DM et al. / Arch Clin Psychiatry. 2019;46(6):151-5

Keywords: Cognition, elderly, frailty, mental health.

\section{Introduction}

Cognitive impairment is becoming more prevalent in older persons, and the consequences of dementia for this population are an increasingly worrying public health issue ${ }^{1}$. Thus, the study of diseases and syndromes most present in the elderly population is critical in order to contribute to the promotion and restoration of their health and quality of life ${ }^{2}$. As well as leading to reduced cognitive capacity and overload of homeostatic control mechanisms, the aging process can also result in frailty ${ }^{3}$.

The frailty syndrome is a complex concept with many definitions and screening methods. It is estimated that the prevalence of the frailty syndrome varies from $5 \%$ to $58 \%{ }^{4}$. Fried et al. ${ }^{5}$ defined frailty as a state of physiological vulnerability associated with aging, which results from a reduced homeostatic reserve and difficulty for the body to adequately respond to stressful events ${ }^{5}$. Thus, clinical manifestations of this syndrome would be linked to impairment of the functional reserve of important organic systems in charge of hormonal, immunological, inflammatory, and neurological processes $^{5}$. Therefore, a frail individual's organism may not develop adaptive responses to stressors, such as intense physical activity, extreme temperatures, or acute diseases ${ }^{5}$. According to the definition by Fried et al. 5 , the frail individual has three or more of the following biological features: unintentional weight loss (greater than $4.5 \mathrm{~kg}$ or $5 \%$ of the body weight in the previous year); self-reported fatigue; muscle weakness; low level of physical activity; and slow walking speed $^{5}$. On the other hand, other definitions of frailty include social, psychological, and cognition factors. Regardless of the diagnostic method, frailty is associated with aggravation of diseases, cognitive and functional decline, hospitalization, and death ${ }^{6}$.
Clinically, cognitive decline progresses in tandem with functional decline ${ }^{7}$. However, the way physical frailty contributes to the cognitive deficit is not fully established in the literature ${ }^{7}$. Scientific evidence suggests that the frailty syndrome can be a significant risk factor for dementia in elderly persons ${ }^{8,9}$, with higher levels of frailty being associated with lower scores of cognitive tests ${ }^{5,10}$. Environmental and genetic factors may also influence the relation between physical frailty and cognitive functions. Moreover, a hypothetical model proposes that oxidative stress, mitochondrial dysfunction, possible damage to DNA, and inflammation may cause impairment in both systems ${ }^{7}$.

The close link between frailty and cognition helped create a new term to describe individuals with both characteristics. Thus, cognitive frailty is a syndrome defined by physical frailty and cognitive impairment in elderly persons without a diagnosis of dementia ${ }^{11}$. Frail elderly persons suffering from cognitive impairment are prone to deleterious effects; consequently, understanding the association between these two variables may contribute to planning early and effective interventions ${ }^{12}$.

A review article examining the current evidence on the relation between frailty and cognition ${ }^{13}$ found that the vast majority of the studies evaluated the frailty syndrome using the components defined by Fried et al. ${ }^{5}$, since the objective was to analyze the physical dimension of the syndrome ${ }^{13}$. As for cognitive evaluation, the studies mainly used brief screening scales, such as the Mini-Mental State Examination (MMSE) ${ }^{13}$. These findings suggest the need to use more complex batteries for cognitive evaluation to determine the domains most altered by frailty.

Interest in how different cognitive domains are altered in the presence of the frailty syndrome is recent. Studies suggest that the memory domain is less affected in frail persons than the executive function ${ }^{14,15}$. On the other hand, a study conducted with 761 elderly persons with no cognitive 
impairment at baseline found that physical frailty was associated with a risk higher than $60 \%$ of developing mild cognitive impairment, and this association was maintained after adjusting for depression and cardiovascular disease symptoms ${ }^{16}$. The frailty syndrome was linked to a faster decline in four cognitive domains (semantic memory, working memory, perceptive speed, and visuospatial capacity) ${ }^{16}$.

A study evaluating 4,317 individuals from the age of 50 found that pre-frailty and frailty are associated with lower scores in the attentional domain in regression analyses ${ }^{17}$. Complementing these data, Han et al. ${ }^{18}$ evaluated 10,388 aged 65 years or older and found that frail elderly had worse scores on the MMSE. The regression analyses showed that cognitive impairment was associated with a higher risk of the syndrome in men, as well as the orientation and attention domains were associated with a higher probability of occurrence of the syndrome in both sexes ${ }^{18}$.

Corroborating the study cited above, Chen et al. ${ }^{19}$ investigated the association between global cognition and frailty in the elderly without dementia. Lower scores on cognitive tests, as well as on their specific domains, were associated with higher likelihood of frailty ${ }^{19}$. These studies reaffirm the close relationship between global cognition, specific cognitive domains, and frailty in the elderly. In this sense, detailed information about both variables can contribute to effective interventions in the clinical context.

In the Brazilian context, few studies have sought to explore the most altered cognitive domains in the presence of the syndrome. To evaluate the frailty syndrome in Brazilian elderly and its related factors, the FIBRA-BR study found that there were more frail and pre-frail elderly with cognitive deficits than elderly classified as non-frail in seven Brazilian cities ${ }^{14,20}$. Components such as muscle strength and slow gait were associated with performance in the MMSE, the verbal fluency test, and the clock drowning test ${ }^{20}$. Besides, the frail elderly showed worse performance in temporal orientation and immediate memory ${ }^{14}$. In this way, continuing to explore the most altered cognitive domains in the presence of frailty through broader batteries of cognitive screening may contribute to uncovering new domains that could be altered in the presence of the syndrome. Thus, the objective of the present study was to investigate the differences in general and domain-specific cognitive performance between non-frail, pre-frail, and frail elderly persons.

\section{Methods}

\section{Setting and participants}

The study was carried out in the city of São Carlos, a municipality located in the interior of the state of São Paulo, Brazil. The city has 28,696 inhabitants with more than 60 years of age, corresponding to $12.9 \%$ of the total population ${ }^{21}$. The study was carried out in a catchment area covered by a family health program, in which 317 individuals aged over 60 years were registered as dwellers. During home visits, 28 individuals were not found in their homes or no longer lived at the address, 5 refused to participate in the study, 2 were bedridden, and 15 did not complete the frailty scale. Thus, the final sample consisted of 267 participants. The data were collected between March 2016 and February 2017.

\section{Procedures}

This study was approved by the ethics committee of the Federal University of São Carlos (CAAE: 48602515.5.0000.5504), and all participants signed the informed consent form prior to participation. Five trained gerontologists conducted the interviews at the participants' homes. Sociodemographic data were also collected.

The participants were divided into frail, pre-frail, and non-frail groups, according to the criteria adopted by Fried et al. ${ }^{5} \mathrm{~A}$ self-report instrument of frailty validated for the Brazilian context was used ${ }^{22}$. The instrument is composed of five biological components: unintentional weight loss, manual grip strength, self-reported fatigue, reduced walking speed, and low level of physical activity22. The classification of frailty follows the criteria of Fried et al.5: "frail" individuals score positively on three or more components, "pre-frail" score positively on one or two components, and "non-frail" do not score positively on any component ${ }^{5,22}$. In this instrument, the elderly respond "yes" or "no" to each one of the biological components: (1) unintentional weight loss - "In the last 12 months, did you lose weight without going on any diet?"; (2) manual grip strength - "In the last 12 months, do you feel weaker or think your strength has decreased?"; (3) reduced walking speed - "Do you think that you are walking more slowly than you did 12 months ago?"; (4) low level of physical activity - "Do you think that you are currently performing less physical activity than you did 12 months ago?", and (5) fatigue - "In the past week, how often did you feel that you could not perform daily activities (you started something but could not finish)?" and "In the past week, how often did the performance of your routine activities require a major effort?" 22

As for the cognitive evaluation, the following instruments were used:

- MMSE: This is a screening tool widely used for evaluation of general cognition with a score ranging from 0 to 30 . It evaluates temporal and spatial orientation, memory (fixation and evocation), language, attention, and calculation ${ }^{23}$;

- Consortium to Establish a Registry for Alzheimer's Disease (CERAD) neuropsychological battery: This battery is composed of the following cognitive tests: verbal fluency (animals), Boston naming test of 15 items, MMSE, word list memory, constructional praxis, delayed word list recall, word list recognition, and delayed praxis recall ${ }^{24}$. In the memory evaluation of this battery, the word list memory, a list of 10 words is presented to the participant, who should remember as many words as possible for a maximum period of 90 seconds (free recall). The procedure is repeated another two times, and the score is calculated as the sum of the words remembered in the three attempts. Subsequently, constructional praxis is evaluated by copying four figures. The delayed recall of the previously presented word list is performed for a maximum of 90 seconds. The 10 initial words are presented along with 10 distracters, and the participant must recognize which words belong to the previously presented list. Finally, the four previously copied drawings are reproduced ${ }^{24}$;

- Brief Cognitive Screening Battery (BCSB): This battery includes verbal fluency (animals), clock drawing test, and figure memory test (incidental, immediate, learning, delayed, and of recognition). It is used with good accuracy in populations with high illiteracy rates or low levels of education ${ }^{25}$. The evaluation of memory of this battery involves the presentation of 10 figures (shoe, spoon, comb, tree, turtle, key, airplane, house, book, and bucket), which should be named aloud immediately by the individual. Then the figures are presented another two times, followed each time by an immediate recall. After performing the verbal fluency test and the clock drawing test, the 10 figures should be remembered in the delayed memory test, followed by a final recognition test (10 target figures with 10 distracting figures) 25 ;

- Subtest of similarity of the Cambridge Mental Disorders Examination of the Elderly (CAMDEX): This test consists of four questions that evaluate the abstraction ability of the participants from the similarity between two things or objects, for instance, "What do an apple and a banana have in common?"26; - Digit span test (backward and forward) of the Wechsler Memory Scale-Revised: Consisting of seven pairs of numerical sequences with different amounts of digits, this test is applied in backward and forward order. In the forward span, the sequences have 3 to 9 numbers, and in the backward span, 2 to 8 numbers ${ }^{27}$. The test ends after error in two consecutive sequences. The maximum quantity of repeated numbers without error is registered for each version ${ }^{27}$.

\section{Statistical analyses}

A descriptive analysis was performed for the independent variables considering the total sample and the three frailty groups (non-frail, 
pre-frail, and frail). Pearson's chi-square test, analysis of variance (ANOVA), or Kruskall-Wallis test were used to assess differences between the groups according to the sample distribution and variable type. The association between frailty and cognitive performance was analyzed using multinomial logistic regression. The relative risk ratio (RRR) and their respective 95\% confidence intervals (IC 95\%) were calculated for each cognitive test, adjusting for age, gender, and education. The "non-frail" group was considered as the reference in the regression models. The analyses were performed using the STATA 15.1 , and the significance level considered was $5 \%$.

\section{Results}

The participants were classified as non-frail $(\mathrm{n}=36)$, pre-frail $(\mathrm{n}=$ $71)$, and frail $(n=160)$. Sociodemographic data (gender, age, and education), as well as the average scores on the cognitive tests in each frailty group, are shown in Table 1 . The frail group had a higher number of women $(63.8 \%)$ than men. The average age was 71.5 years old ( \pm 8.6$)$, and the sample had low levels of schooling (63.8\% with less than 4 years of schooling). The frail group presented a lower mean in all cognitive test scores. Nevertheless, the tests with statistically significantly differences were the MMSE, the CERAD battery word list, and the BCSB recognition item, the Boston naming test, and the backward digit span test.

The multinomial logistic regression results are shown in Table 2. Frailty was significantly associated with lower scores on the MMSE $(\mathrm{RRR}=0.86$; IC 95\% 0.78-0.96; $\mathrm{p}<0.01)$, CERAD word list memory $(\mathrm{RRR}=0.92 ;$ IC 95\% 0.86-0.99; $\mathrm{p}=0.02)$, and BCSB figure list recognition $(R R R=0.78$; IC 95\% 0.62-0.99; $p=0.04)$. The pre-frail level was associated with lower scores on the CERAD word list memory $(\mathrm{RRR}=0.93$; IC 95\% 0.86-1.00; $\mathrm{p}=0.04)$ and Boston naming test $(\mathrm{RRR}=0.82 ;$ IC 95\% 0.69-0.99; $\mathrm{p}=0.03)$.

Table 1. Sociodemographic variables and battery of cognitive tests in the three groups of frailty (non-frail, pre-frail and frail)

\begin{tabular}{|c|c|c|c|c|c|}
\hline Variable & Total $(n=267)$ & Non-frail $(n=36)$ & Pre-frail $(n=71)$ & Frail $(n=160)$ & $p$ \\
\hline Age & $70.2( \pm 7.7)$ & $68.0( \pm 5.9)$ & $68.6( \pm 5.5)$ & $71.5( \pm 8.6)$ & 0.04 \\
\hline $\begin{array}{r}\text { Sex } \\
F \\
M\end{array}$ & $\begin{array}{l}61 \% \\
39 \%\end{array}$ & $\begin{array}{l}36.1 \% \\
63.9 \% \\
\end{array}$ & $\begin{array}{l}60.6 \% \\
39.4 \% \\
\end{array}$ & $\begin{array}{l}63.8 \% \\
36.3 \% \\
\end{array}$ & $<0.01$ \\
\hline $\begin{array}{c}\text { Education } \\
\geq 4 \text { year } \\
<4 \text { year }\end{array}$ & $\begin{array}{l}43.8 \% \\
56.2 \% \\
\end{array}$ & $\begin{array}{l}52.8 \% \\
47.2 \%\end{array}$ & $\begin{array}{l}56.3 \% \\
43.7 \%\end{array}$ & $\begin{array}{l}36.3 \% \\
63.8 \%\end{array}$ & $<0.01$ \\
\hline MMSE & $22.2( \pm 5.0)$ & $24.6( \pm 4.0)$ & $23.0( \pm 4.7)$ & $21.2( \pm 5.1)$ & $<0.01$ \\
\hline Word list memory (CERAD) & $12.1(5.8)$ & $14.7( \pm 6.0)$ & $12.5( \pm 5.4)$ & $11.5( \pm 5.8)$ & $<0.01$ \\
\hline Delayed recall (CERAD) & $3.0( \pm 2.5)$ & $3.5( \pm 2.4)$ & $3.1( \pm 2.5)$ & $2.9( \pm 2.5)$ & 0.32 \\
\hline Recognition (CERAD) & $7.2( \pm 3.1)$ & $7.9( \pm 3.2)$ & $7.3( \pm 3.0)$ & $7.0( \pm 3.0)$ & 0.07 \\
\hline Constructional praxis (CERAD) & $5.3( \pm 3.3)$ & $5.7( \pm 3.4)$ & $5.8( \pm 3.0)$ & $5.0( \pm 3.4)$ & 0.30 \\
\hline Figures list memory (BCSB) & $19.8(6.3)$ & $20.2( \pm 5.8)$ & $20.9( \pm 6.3)$ & $19.3( \pm 6.4)$ & 0.10 \\
\hline BCSB (Delayed recall) & $6.5( \pm 2.7)$ & $6.5( \pm 2.3)$ & $7.0( \pm 2.8)$ & $6.3( \pm 2.8)$ & $<0.01$ \\
\hline BCSB (Recognition) & $8.4( \pm 2.7)$ & $9.3( \pm 1.6)$ & $8.5( \pm 2.6)$ & $8.1( \pm 2.8)$ & $<0.01$ \\
\hline Abstraction subtest (CAMDEX) & $2.7( \pm 2.2)$ & $3.3( \pm 2.6)$ & $2.8( \pm 2.0)$ & $2.4( \pm 2.2)$ & 0.11 \\
\hline Clock Drawing Test & $4.8( \pm 3.6)$ & $5.0( \pm 3.5)$ & $5.2( \pm 3.6)$ & $4.7( \pm 3.6)$ & 0.32 \\
\hline Verbal fluency & $10.4( \pm 4.0)$ & $10.9( \pm 4.4)$ & $10.7( \pm 4.1)$ & $10.1( \pm 3.7)$ & 0.68 \\
\hline Boston Naming test & $11.2( \pm 2.9)$ & $12.4( \pm 2.3)$ & $11.0( \pm 3.0)$ & $11.0( \pm 2.8)$ & 0.04 \\
\hline Digit extension test (Forward) & $4.3( \pm 1.4)$ & $4.5( \pm 1.2)$ & $4.6( \pm 1.4)$ & $4.2( \pm 1.4)$ & 0.14 \\
\hline Digit extension test (Backward) & $2.3( \pm 1.2)$ & $2.7( \pm 0.9)$ & $2.4( \pm 1.1)$ & $2.1( \pm 1.3)$ & 0.03 \\
\hline
\end{tabular}

MMSE: Mini-Mental State Examination; CERAD: Consortium to Establish a Registry for Alzheimer's disease neuropsychological battery; BCSB: Brief Cognitive Screening Battery.

Table 2. Multivariate regression analysis of cognitive domains in relation to frailty $(n=267)$

\begin{tabular}{|c|c|c|c|c|}
\hline \multirow{3}{*}{$\begin{array}{l}\text { Variable } \\
\text { Reference group } \\
\text { (non-frail) }\end{array}$} & \multicolumn{4}{|c|}{ Groups } \\
\hline & \multicolumn{2}{|c|}{ Pre-frail } & \multicolumn{2}{|c|}{ Frail } \\
\hline & RRR & $p$ & RRR & $p$ \\
\hline MMSE & $0.90(0.80-1.00)$ & 0.06 & $0.86(0.78-0.96)$ & $<0.01$ \\
\hline Word list memory (CERAD) & $0.93(0.86-1.00)$ & 0.04 & $0.92(0.86-0.99)$ & 0.02 \\
\hline Delayed recall (CERAD) & $0.91(0.76-1.07)$ & 0.25 & $0.92(0.79-1.07)$ & 0.28 \\
\hline Recognition (CERAD) & $0.91(0.77-1.06)$ & 0.22 & $0.93(0.81-1.08)$ & 0.35 \\
\hline Constructional praxis (CERAD) & $1.01(0.88-1.17)$ & 0.84 & $1.00(0.87-1.13)$ & 0.91 \\
\hline Figures list memory (BCSB) & 1.01 (0.94-1.09) & 0.78 & $0.98(0.92-1.05)$ & 0.67 \\
\hline Delayed recall (BCSB) & $1.03(0.88-1.22)$ & 0.69 & $0.97(0.84-1.13)$ & 0.70 \\
\hline Recognition (BCSB) & $0.79(0.62-1.01)$ & 0.06 & $0.78(0.62-0.99)$ & 0.04 \\
\hline Abstraction subtest (CAMDEX) & $0.87(0.72-1.06)$ & 0.17 & $0.88(0.73-1.05)$ & 0.15 \\
\hline Clock Drawing Test & $1.00(0.88-1.14)$ & 0.93 & $1.02(0.90-1.15)$ & 0.75 \\
\hline Verbal fluency & $1.00(0.90-1.10)$ & 0.93 & $0.99(0.90-1.09)$ & 0.81 \\
\hline Boston Naming test & $0.82(0.69-0.99)$ & 0.03 & $0.89(0.75-1.06)$ & 0.19 \\
\hline Digit extension test (Forward) & $1.15(0.84-1.56)$ & 0.39 & $1.01(0.76-1.34)$ & 0.94 \\
\hline Digit extension test (Backward) & $0.89(0.61-1.31)$ & 0.57 & $0.80(0.57-1.13)$ & 0.21 \\
\hline
\end{tabular}

Adjusted for age, sex and education. RRR: Relative Risk Ratio; MMSE: Mini-Mental State Examination; CERAD: Consortium to Establish a Registry for Alzheimer's disease neuropsychological battery; BCSB: Brief Cognitive Screening Battery. 


\section{Discussion}

In the present study, most participants were classified as frail (59.9\%) and pre-frail $(26.6 \%)$. Participants in the frail group were more likely to be women and have advanced age and low education level. This result is compatible with those from other studies evaluating the frailty syndrome in elderly persons 28,29 . This profile of frail elderly persons may be explained by the feminization of ageing and a significant physiological loss following this process, which may contribute to the emergence of the frailty syndrome in this population ${ }^{5}$. Furthermore, although low schooling is not directly considered as a risk factor for frailty, it can consequently decrease the quality of life of aging individuals, thus influencing the occurrence of the syndrome ${ }^{30}$.

A review of the literature examining the mechanisms involved in the relation between the frailty syndrome and cognitive performance found that few studies tried to answer in an experimental way the effect of the frailty syndrome on the decline of cognitive functions ${ }^{31}$. However, longitudinal studies indicate that frailty and cognitive decline are mutually related, since the presence of frailty increases the risk of cognitive decline and the presence of cognitive decline increases the risk of frailty. A meaningful discussion to be held is the role of cognitive decline in the frailty syndrome. Sternberg et al. ${ }^{4}$ found that the cognitive domain was present in $50 \%$ of the syndrome definitions ${ }^{4}$. Thus, the central aspect of this debate lies in the frailty construct.

In this study, the biological model proposed by Fried et al. ${ }^{5}$ was used to evaluate the association of frailty with cognitive aspects, since using a model that included cognition could erroneously increase the association because of collinearity between the variables. Moreover, the model by Fried et al. ${ }^{5}$ is the most frequently used in the current literature. However, it must be pointed out that other frailty models include cognition among the possible domains that may be altered in the syndrome. This study found significant differences between the frail and non-frail groups in global cognition, BCSB figure recognition, and CERAD word list. Additionally, we found differences between the non-frail and pre-frail groups in the Boston naming test. The tests used to evaluate these domains are traditionally used in the neuropsychological evaluation of dementias, especially Alzheimer's disease (AD). Buchman et al. ${ }^{32}$ examined the relation between frailty, cerebral infarcts, dementia with Lewy bodies, and $\mathrm{AD}$, and found that the frailty syndrome was associated only with $\mathrm{AD}(\beta=0.252$, $\mathrm{SE}=0.077, \mathrm{p}=0.001)^{32}$. The authors raised the hypothesis that the frailty syndrome could be a non-cognitive manifestation of $\mathrm{AD}^{32}$. In this context, the introduction of frailty assessment in individuals with dementia, especially those with the $\mathrm{AD}$, seems fundamental. Research and public policies should increasingly focus on developing strategies to maintain cognitive and physical health during the aging process.

Bunce et al. ${ }^{33}$ assessed associations between specific cognitive domains and the frailty syndrome ${ }^{33}$. At baseline, the frail elderly persons showed poorer cognitive performance in processing speed, word and face recognition, episodic memory, verbal fluency, and reaction time ${ }^{33}$. However, there were no significant changes throughout the 12 years of study for any of the cognitive variables ${ }^{33}$. Our findings also showed a higher association between the memory domain and frailty, so that each figure that was recognized in the $\mathrm{BCSB}$ decreased the risk of the elderly person being in the frail group by $22 \%$, just as each word remembered in the list reduced the risk by $8 \%$.

In addition, we found that the Boston naming test was more altered in pre-frail elderly persons. Since pre-frailty is an intermediate state of the syndrome, we hypothesized that language impairment could contribute to a more rapid impairment of other cognitive domains and, consequently, the progression of the syndrome. However, we did not find any studies specifically analyzing the language domain in the presence of frailty. Thus, our findings point to a cognitive domain that may also be altered in the presence of the syndrome and call for new studies assessing the relationship between these two variables more specifically.
Another study that explored the relation between frailty and functioning in cognitive domains found significant differences when comparing frail and non-frail elderly in executive functions and processing speed, but no significant differences in global cognition ${ }^{15}$. When comparing this finding with our results, the methodological differences of the studies may explain the differences found in cognitive domains. For example, the methods for screening the syndrome and defining the frailty groups were different in the two studies. The authors of the former study also prioritized instruments that assessed processing speed and executive functions, e.g., the Wechsler Adult Intelligence Scale (WAIS-III), the Trail Making Test (part A), and the Stroop color-word test ${ }^{15}$.

In an attempt to explore the frailty and cognitive performance of Brazilian elderly through the BCSB, Yassuda et al. ${ }^{20}$ found an association of gait speed and reduced strength to cognitive test performance in regression analysis ${ }^{20}$. In this sense, the evidence suggests that impaired motor skills may be related to cognitive impairment. Sternäng et al. ${ }^{34}$ examined the relationship between grip strength and cognitive performance by assessing 708 participants over 20 years. The authors used instruments that assessed verbal skills, spatial abilities, processing speed and memory ${ }^{34}$. The results evidenced an association between decreased grip strength and decreased performance in all cognitive tests ${ }^{34}$. The evidence presented here along with the results found in our study reaffirm that motor and cognitive abilities share common mechanisms. Thus, new studies should explore these variables more specifically.

The present study has some limitations. Firstly, more instruments assessing the memory domain were used, and this choice may have contributed to the obtained results since more specific tests to assess other domains, mainly functions related to the frontal lobe, may be essential to detect differences between the groups. Secondly, other potential confounders such as alcohol use and smoking were not considered in the analyzes and individuals with dementia, Parkinson's disease, or severe depression were not excluded from the final sample. Lastly, the cross-sectional study does not allow to establish causal relationships between the frailty syndrome and decline in the cognitive domains evaluated, which would be possible in longitudinal designs.

\section{Conclusion}

The prevalence of frailty syndrome is a phenomenon that deserves attention from health professionals and researchers. In this study, we found a large number of elderly individuals classified as frail according to their self-perception. Moreover, we found an association between frailty and poor global cognitive performance, memory, learning, and language. New prospective studies are fundamental to evaluate the relation between frailty and cognitive functioning in specific domains, especially those related to the frontal lobe.

\section{Acknowledgment}

This study was financed in part by the Coordenação de Aperfeiçoamento de Pessoal de Nível Superior - Brasil (Capes) - Finance Code 001; and by the Brazilian fostering agency Fapesp (grant \#: 2015/16412-1). Daiene de Morais is a recipient of a scholarship from the National Council for Scientific and Technological Development/CNPq, Brazil (grant: \# 132257/2017-3).

\section{Disclosure}

We have no conflict of interest to declare.

\section{References}

1. Wang C, Ji X, Wu X, Tang Z, Zhang X, Guan S, et al. Frailty in relation to the risk of Alzheimer's disease, dementia, and death in older Chinese adults: A seven-year prospective study. J Nutr Health Aging. 2017;21(6):648-54. 
2. Ng TP, Ling LHA, Feng L, Nyunt MSZ, Feng L, Niti M, et al. Cognitive effects of multi-domain interventions among pre-frail and frail community-living older persons: randomized controlled trial. J Gerontol A Biol Sci Med Sci. 2018;73(6):806-12.

3. Panza F, Seripa D, Solfrizzi V, Tortelli R, Greco A, Pilotto A, et al. Targeting cognitive frailty: clinical and neurobiological roadmap for a single complex phenotype. J Alzheimers Dis. 2015;47(4):793-813.

4. Sternberg SA, Schwartz AW, Karunananthan S, Bergman H, Mark Clarfield A. The identification of frailty: a systematic literature review. J Am Geriatr Soc. 2011;59(11):2129-38.

5. Fried LP, Tangen CM, Walston J, Newman AB, Hirsch C, Gottdiener J, et al.; Cardiovascular Health Study Collaborative Research Group. Frailty in older adults: evidence for a phenotype. J Gerontol A Biol Sci Med Sci. 2001;56(3):M146-57.

6. Rolfson DB, Majumdar SR, Tsuyuki RT, Tahir A, Rockwood K. Validity and reliability of the Edmonton Frail Scale. Age Ageing. 2006;35(5):526-9.

7. Halil M, Kizilarslanoglu MC, Kuyumcu ME, Yesil Y, Jentoft AC. Cognitive aspects of frailty: mechanisms behind the link between frailty and cognitive impairment. J Nutr Health Aging. 2015;19(3):276-83.

8. Song X, Mitnitski A, Rockwood K. Age-related deficit accumulation and the risk of late-life dementia. Alzheimers Res Ther. 2014;6(5-8):54.

9. Searle SD, Rockwood K. Frailty and the risk of cognitive impairment. Alzheimers Res Ther. 2015;7(1):54.

10. Neri AL, Yassuda MS, Araújo LFD, Eulálio MDC, Cabral BE, Siqueira MECD, et al. Metodologia e perfil sociodemográfico, cognitivo e de fragilidade de idosos comunitários de sete cidades brasileiras: Estudo FIBRA. Cad Saúde Pública. 2013;29(4):778-92.

11. Kelaiditi E, Cesari M, Canevelli M, Van Kan GA, Ousset PJ, Gillette-Guyonnet S, et al.; IANA/IAGG. Cognitive frailty: rational and definition from an (I.A.N.A./I.A.G.G.) international consensus group. J Nutr Health Aging. 2013;17(9):726-34.

12. Butler A, Gallagher D, Gillespie P, Crosby L, Ryan D, Lacey L, et al. Frailty: a costly phenomenon in caring for elders with cognitive impairment. Int J Geriatr Psychiatry. 2016;31(2):161-8.

13. Canevelli M, Cesari M, Vankan GA. Frailty and cognitive decline: how do they relate? Curr Opin Clin Nutr Metab Care. 2015;18(1):43-50.

14. Macuco CRM, Batistoni SST, Lopes A, Cachioni M, da Silva Falcão DV, Neri AL, et al. Mini-Mental State Examination performance in frail, pre-frail, and non-frail community dwelling older adults in Ermelino Matarazzo, São Paulo, Brazil. Int Psychogeriatrics. 2012;24(11):1725-31.

15. Langlois F, Vu TTM, Kergoat MJ, Chassé K, Dupuis G, Bherer L. The multiple dimensions of frailty: physical capacity, cognition, and quality of life. Int Psychogeriatrics. 2012;24(9):1429-36.

16. Boyle PA, Buchman AS, Wilson RS, Leurgans SE, Bennett DA. Physical frailty is associated with incident mild cognitive impairment in community-based older persons. J Am Getriatr Soc. 2010;58(2):248-55.

17. O'Halloran AM, Finucane C, Savva GM, Robertson IH, Kenny RA. Sustained attention and frailty in the older adult population. J Gerontol B Psychol Sci Soc Sci. 2013;69(2):147-56.

18. Han ES, Lee Y, Kim J. Association of cognitive impairment with frailty in community-dwelling older adults. Int Psychogeriatrics. 2014;26(1):155-63.
19. Chen S, Honda T, Narazaki K, Chen T, Nofuji Y, Kumagai S. Global cognitive performance and frailty in non-demented community-dwelling older adults: findings from the Sasaguri Genkimon Study. Geriatr Gerontol Int. 2016;16(6):729-36.

20. Yassuda MS, Lopes A, Cachioni M, Falcao DVS, Batistoni SST, Guimaraes VV, et al. Frailty criteria and cognitive performance are related: Data from the FIBRA study in Ermelino Matarazzo, São Paulo, Brazil. J Nutr Health Aging. 2012;16(1):55-61.

21. Instituto Brasileiro de Geografia e Estatística (IBGE). Censo 2010. Disponível em: https://censo2010.ibge.gov.br/sinopse/index. php? dados=26\&uf=35\#topo_piramide. Acesso em: 3 maio 2018.

22. Nunes DP, Duarte YADO, Santos JLF, Lebrão ML. Screening for frailty in older adults using a self-reported instrument. Rev Saúde Pública. 2015;49(2).

23. Folstein MF, Folstein SE, McHugh PR. "Mini-mental state": a practical method for grading the cognitive state of patients for the clinician. J Psychiatr Res. 1975;12(3):189-98.

24. Bertolucci PHF, Okamoto IH, Brucki SMD, Siviero MO, Toniolo Neto J, Ramos LR. Applicability of the CERAD neuropsychological battery to Brazilian elderly. Arq Neuro-psiquiatr. 2001;59(3):532-6.

25. Nitrini R, Caramelli P, Herrera E, Porto CS, Charchat-Fichman H, Carthery MT, et al. Performance of illiterate and literate nondemented elderly subjects in two tests of long-term memory. J Int Neuropsychol Soc. 2004;10(4):634-8.

26. Roth M, Tym E, Mountjoy CQ, Huppert FA, Hendrie H, Verma S, et al. CAMDEX: a standardised instrument for the diagnosis of mental disorder in the elderly with special reference to the early detection of dementia. Br J Psychiatry. 1986;149(6):698-709.

27. Roth MTYME, Tym E, Mountjoy CQ, Huppert FA, Hendrie H, Verma S, Goddard R. A compendium of neuropsychological tests: administration, norms, and commentary. American Chemical Society; 2006.

28. Ge L, Yap CW, Heng BH. Prevalence of frailty and its association with depressive symptoms among older adults in Singapore. Aging Ment Health. 2019;23(3):319-24.

29. Nóbrega PVDN, Maciel ÁCC, De Almeida Holanda CM, Oliveira Guerra $\mathrm{R}$, Araújo JF. Sleep and frailty syndrome in elderly residents of long-stay institutions: a cross-sectional study. Geriatr Gerontol Int. 2014;14(3):605-12.

30. Franse CB, Van Grieken A, Qin, L, Melis RJ, Rietjens JA, Raat H. Socioeconomic inequalities in frailty and frailty components among community-dwelling older citizens. PloS One. 2017;12(11):e0187946.

31. Robertson DA, Savva GM, Kenny RA. Frailty and cognitive impairment - a review of the evidence and causal mechanisms. Ageing Res Rev. 2013;12(4):840-51.

32. Buchman AS, Schneider JA, Leurgans S, Bennett DA. Physical frailty in older persons is associated with Alzheimer disease pathology. Neurology. 2008;71(7):499-504.

33. Bunce D, Batterham PJ, Mackinnon AJ. Long-term associations between physical frailty and performance in specific cognitive domains. J Gerontol B Psychol Sci Soc Sci. 2019;74(6):919-26.

34. Sternäng O, Reynolds CA, Finkel D, Ernsth-Bravell M, Pedersen NL, Dahl Aslan AK. Grip strength and cognitive abilities: associations in old age. J Gerontol B Psychol Sci Soc Sci. 2016;71(5):841-8. 